\title{
Structure and energetics of the $\mathrm{Si}_{-} \mathrm{SiO}_{2}$ interface
}

\author{
Yuhai Tu and J. Tersoff \\ IBM Research Division, T. J. Watson Research Center \\ P. O. Box 218, Yorktown Heights, NY 10598
}

(June 26, 2021)

\begin{abstract}
Silicon has long been synonymous with semiconductor technology. This unique role is due largely to the remarkable properties of the $\mathrm{Si}-\mathrm{SiO}_{2}$ interface, especially the (001)-oriented interface used in most devices [1]. Although $\mathrm{Si}$ is crystalline and the oxide is amorphous, the interface is essentially perfect, with an extremely low density of dangling bonds or other electrically active defects. With the continual decrease of device size, the nanoscale structure of the silicon/oxide interface becomes more and more important. Yet despite its essential role, the atomic structure of this interface is still unclear. Using a novel Monte Carlo approach, we identify low-energy structures for the interface. The optimal structure found consists of Si-O-Si "bridges" ordered in a stripe pattern, with very low energy. This structure explains several puzzling experimental observations.

Experiments offer many clues to the interface structure, but their interpretation remains controversial, because of the complexities inherent in studying disordered materials. Proposed models range from a graded interface [2, 3] to a sharp interface [4] and even to a crystalline oxide layer at the interface [5]. Most theoretical studies have involved guessing reasonable structures [6], sometimes even using hand-built models [7]. More recently, there have been attempts to obtain an unbiased structure using unconstrained molecular dynamics (MD) [8] and Monte Carlo (MC) studies [9]. However, because of kinetic limitations these studies have not been able to identify the equilibrium structure. Calculations of the interface energy are also not possible with existing methods.
\end{abstract}


Here we employ a novel approach in which the $\mathrm{Si}_{-} \mathrm{SiO}_{2}$ system is modeled as a continuous network of bonds connecting the atoms, and the thermodynamic ensemble of possible network topologies is explored via Monte Carlo (MC) sampling. The basic method has been described elsewhere [10] in a simpler context. But the present application demonstrates that this approach makes possible an entirely new class of computational studies of disordered systems.

Our approach samples only defect-free configurations, in which Si and O have four and two bonds respectively, and there are no O-O bonds. Because of this restriction, the energy may be reasonably approximated by a valence-force model:

$$
\begin{aligned}
E_{\{r\}}= & \frac{1}{2} \sum_{i} k_{b}\left(b_{i}-b_{0}\right)^{2} \\
& +\frac{1}{2} \sum_{i, j} k_{\theta}\left(\cos \theta_{i j}-\cos \theta_{0}\right)^{2} .
\end{aligned}
$$

Here $\{\mathbf{r}\}$ is the set of atom positions, $E_{\{r\}}$ is the total energy for a given network topology and given $\{\mathbf{r}\}$. $i$ represents the $i$ th bond, $b_{i}$ is ts length, $\theta_{i j}$ is the angle between bonds $i$ and $j$ connected to a common atom. The material parameters depend implicitly on the type of atom, where $b_{0}$ is the preferred bond length, $\theta_{0}$ is the preferred bond angle, and $k_{\theta}$ and $k_{b}$ are "spring constants." We take $k_{b, \mathrm{Si}-\mathrm{Si}}=9.08 \mathrm{eV} / \stackrel{\circ}{ }^{2}, k_{\theta, \mathrm{Si}-\mathrm{Si}-\mathrm{Si}}=3.58 \mathrm{eV}, b_{0, \mathrm{Si}-\mathrm{Si}}=2.35 \AA$, $\cos \left(\theta_{0, \mathrm{Si}}\right)=-1 / 3, k_{b, \mathrm{Si}-\mathrm{O}}=27.0 \mathrm{eV}, b_{0, \mathrm{Si}-\mathrm{O}}=1.6 \stackrel{\circ}{\circ}, k_{\theta, \mathrm{O}-\mathrm{Si}-\mathrm{O}}=4.32 \mathrm{eV}$ and $k_{\theta, \mathrm{Si}-\mathrm{O}-\mathrm{Si}}=0.75 \mathrm{eV}$, and $\cos \left(\theta_{0, \mathrm{O}}\right)=-1$. For Si-Si-O bonds we set the spring constant to be the geometric mean $k_{\theta, \mathrm{Si}-\mathrm{Si}-\mathrm{O}}=\left(k_{\theta, \mathrm{Si}-\mathrm{Si}-\mathrm{Si}} k_{\theta, \mathrm{O}-\mathrm{Si}-\mathrm{O}}\right)^{1 / 2}$. (There is an additional term in the energy which simply enforces the restriction of two and four neighbors for $\mathrm{O}$ and $\mathrm{Si}$, respectively [10].)

In order to focus on the role of network structure, we treat the energy as a function solely of bond topology, minimizing $E_{\{r\}}$ with respect to the geometrical coordinates $\{\mathbf{r}\}$. Thus for a given network topology

$$
E=\min _{\{\mathbf{r}\}} E_{\{r\}}
$$

The structure of the system is allowed to evolve toward thermodynamic equilibrium through Monte-Carlo bond-switching moves [10,11]. (We adapt the original move to preclude O-O 
bonds.) At each step a random trial bond-switch is accepted with probability $e^{-\Delta E / k T}$ (or 1 for energy-lowering moves), guaranteeing that the system will evolve toward thermodynamic equilibrium. This approach gives a fairly accurate description of the structure of both amorphous $\mathrm{Si}$ [10,11] and amorphous $\mathrm{SiO}_{2}$. Specifically, we have verified that the average bondlength and bond angles are in agreement with experiment, and the elastic constants are accurate to better than $20 \%$.

Our model for the energy is rather simple compared with the more accurate ab initio methods used in some recent studies [6, 8, 9]. Since the $\mathrm{Si}_{-} \mathrm{SiO}_{2}$ system is dominated by steric constraints, our approach should nevertheless be reasonably accurate for the defectfree structures considered here. More important, it allows the large-scale MC sampling necessary for the system to move toward thermodynamic equilibrium, which is not feasible with $a b$ initio methods. It also allows us to determine the actual interface energy, using thermodynamic averaging. More accurate methods are not at present able to determine the interface energy, even for a given interface structure, because it is impractical to average over the statistical ensemble of configurations of the amorphous oxide.

We begin with a convenient though unphysical structure, a perfect interface between crystalline $\mathrm{Si}$ and highly strained $\alpha$-crystobalite. We use 10 layers of $\mathrm{Si}$, and $\mathrm{SiO}_{2}$ containing an equal number of Si atoms, periodically repeated in the interface-normal (z) direction. In the other two dimension, we use cells with $2 \times 2$ and $4 \times 4$ periodicity, for a total of 160 and 640 atoms respectively. To accurately describe an oxide at a free $\mathrm{Si}$ surface, the cell size is constrained to match $\mathrm{Si}(001)$ in two directions, while the period normal to the interface is allowed to vary to maintain zero stress in that direction.

We first perform MC bond-switching within the oxide, allowing it to amorphize and to relax the large strain by viscous flow. We then perform unconstrained MC switching of the entire system, allowing it to equilibrate for up to a total of 300,000 MC steps. To accelerate the evolution, the MC "temperature" used is quite high, $2600^{\circ} \mathrm{C}$; but this refers only to the degree of disorder allowed in the network topology [10]. 
We have carried out 10 independent MC simulations for a $2 \times 2$ cell. The resulting interface structures are shown in Fig. 1. The key structural element is an oxygen bridge between each pair of Si atoms terminating the Si crystal. This eliminates half the bonds from the Si side, correcting the mismatch between the bond densities in the two very different materials. This structure allows each atom to maintain its preferred coordination, with essentially no additional distortion of the bond angles or bond length beyond that already present due to the amorphous nature of the oxide. Bridge bonds have appeared in several previous models of the $\mathrm{Si}_{-} \mathrm{SiO}_{2}$ interface [5, [7,9]. However, it has apparently not been previously recognized that these are the key element, giving an ideal low-energy interface.

All 10 simulations gave fully bridge-bonded structures. However, two distinct arrangements are possible within our $2 \times 2$ periodicity, and both occur in the simulations. We refer to these as the "stripe" and "check" phases, respectively, and they are compared in Fig. 1. We have obtained the same structures using a somewhat different energy function [12], showing that it does not depend on the precise parameter values used.

Similar runs with a $4 \times 4$ cell also give bridge-bonded structures, but the system typically becomes "stuck" in a metastable state with incomplete (of order 75\%) bridge bonding. The energy is consistently lower in structures with more complete bridge bonding. The key role of the bridge bonds is illustrated in Fig. 2, where the total energy of the system and the number of bridge bonds at the interface are plotted against MC "time" for a typical $4 \times 4$ simulation. There is a clear drop in energy each time a new bridge bond is formed. A fully bridge-bonded structure has the lowest energy and is stable under annealing. Thus it seems clear that, with sufficiently long annealing, the $4 \times 4$ cell would always reach the ideal stripe structure. A side view of this structure is shown in Fig. 3a.

In equilibrium, the actual interface structure is that which minimizes the interface energy (or more precisely, the free energy). The interface energy can be calculated by subtracting the bulk energy of the amorphous oxide and crystalline Si (obtained in independent calculations) from the total energy. In all cases the energy is averaged over roughly 10,000 MC steps after the system reaches equilibrium. For the stripe phase, the calculated interface energy is 
$6.8 \pm 1.3 \mathrm{meV} / \AA^{2}(0.10 \mathrm{eV}$ per $1 \times 1$ cell $)$, an order of magnitude smaller than the energy of a free Si surface. For the check phase, we find a slightly higher energy of $9.5 \pm 1.9 \mathrm{meV} / \AA^{2}$ $(0.14 \mathrm{eV}$ per $1 \times 1$ cell) .

We can gain further insight into the energetics by decomposing the total energy of the system into individual atomic contributions. This decomposition is not unique, but a natural choice is to divide the bond-stretching energy in Eq. 1 equally between the two atoms. Half of the bond-angle energy is assigned to the vertex atom, and one quarter to each of the other atoms.

In Fig. 3b, the energy of each atom is plotted versus the $\mathrm{z}$ coordinate, for the low-energy $4 \times 4$ stripe structure after equilibration for $300,000 \mathrm{MC}$ steps. A striking feature is that the main contribution to the interface energy comes from local distortions inside the crystalline Si. The energy within the oxide is rather uniform, even right up to the interface.

There has been considerable interest in the possibility of a crystalline interfacial oxide [5.13]. We can form an interface between $\mathrm{Si}(001)$ and tridymite (0001) which resembles the stripe phase above, but the tridymite is under considerable strain (about $7 \%$ in one direction and $13 \%$ in the other). The properties of this interface are summarized in Fig. 4 . The interface energy is actually much higher than that for amorphous $\mathrm{SiO}_{2}$, about $29 \mathrm{meV} / \AA^{2}$ (0.43 eV per $1 \times 1$ cell). Thus there appears to be no interfacial driving force for formation of a crystalline oxide.

Yet several experiments have suggested the presence of a crystalline oxide layer roughly $5 \AA$ thick at the interface, based on both electron microscopy [5] and x-ray diffraction [13]. These results have remained an outstanding puzzle, but they are immediately explained by our structure.

Electron microscopy suggested a $5 \AA$ layer of tridymite at the interface [5]. Comparison of Fig. 3a and Fig. 4a shows that the structure of the Si-tridymite interface is indistinguishable from the more realistic crystal-amorphous interface, in a region several angstroms thick at the interface. Thus our proposed interface structure is entirely consistent with the electron 
microscopy results. However, it is best viewed as an ordered interface structure, without reference to any crystalline bulk phase. In no case did we see evidence for an ordered phase extending further into the oxide.

X-ray diffraction experiments show an ordered $2 \times 1$ structure at the interface, with a thickness of under $6 \AA$ and a domain size comparable to the step spacing [13. The stripe phase exactly satisfies these characteristics. It has an overall $2 \times 1$ periodicity. Moreover, every interface step causes a $90^{\circ}$ rotation from $2 \times 1$ to $1 \times 2$, so the step spacing sets an upper bound on the domain size. The presence of random small atomic displacements, reflected in Figs. 1 and 3, explains the inability of Ref. [13] to determine precise atomic positions from the diffraction data.

Finally we note that in several experiments, photoemission has been used to measure the number of Si atoms at the interface having intermediate oxidation states [2,3,14]. Many theoretical studies have attempted to reproduce or explain these statistics [6,9], but the interpretation is surprisingly subtle [14]. Nevertheless, there appears to be some concensus that the primary connection between $\mathrm{Si}$ and $\mathrm{SiO}_{2}$ occurs via $\mathrm{Si}^{+2}$ [3, 14], as in our model.

In conclusion, we have identified a simple, defect-free, ordered structure for the $\mathrm{Si}-\mathrm{SiO}_{2}$ interface. It has low energy, and appears to reconcile the various puzzling experimental observations. The computational method focuses on a more complete exploration of the thermodynamic ensemble, even when this requires significant approximations in treating the energetics. It is our hope that this approach will open the door to a new class of computation studies of disordered systems. 


\section{REFERENCES}

[1] Feldman, L. C., Gusev, E. P. and Garfunkel, E. in Fundamental Aspects of ultrathin dielectrics on Si-based devices (eds Garfunkel, E., Gusev, E. and Vul' A.) 1-24 (Kluwer Academic Publishers, Boston, 1998) and references therein.

[2] Himpsel, F. J., McFeely, F. R., Taleb-Ibrahimii, A., Yarmoff, J. A., and Hollinger, G. Microscopic structure of the $\mathrm{SiO}_{2} / \mathrm{Si}$ interface. Phys. Rev. B 38, 6084 (1988).

[3] Grunthaner, P. J., Hecht, M. H., and Johnson, N. M. The localization and crystallographic dependence of $\mathrm{Si}$ suboxide species at the $\mathrm{SiO}_{2} / \mathrm{Si}$ interface. J. Appl. Phys. 61, 629 (1987).

[4] Luh D. A., Miller T., and Chiang T. C. Statistical Cross-Linking at the $\mathrm{Si}(111) / \mathrm{SiO}_{2}$ interface. Phys. Rev. Lett. 79, 3014 (1997).

[5] Ourmazd, A., Taylor, D. W., Rentschler, J. A., and Bevk, J. Si $\rightarrow \mathrm{SiO}_{2}$ Transformation: Interfacial Structure and Mechanism. Phys. Rev. Lett. 59, 213 (1987).

[6] Pasquarello, A., Hybertsen, M. S. and Car, R. Structurally relaxed models of the $\mathrm{Si}(001)-\mathrm{SiO}_{2}$ interface. Appl. Phys. Lett. 68, 625 (1996).

[7] Ohdomari, I., Akatsu, H., Yamakoshi, Y., and Kishimoto, K. The structural models of the $\mathrm{Si} / \mathrm{SiO}_{2}$ Interface. J. Non-Crst. Solids 89, 239 (1987).

[8] Pasquarello, A., Hybertsen, M. S. and Car, R., Interface structure between silicon and its oxide by first-princeples molecular dynamics. Nature 396, 58 (1998).

[9] Ng, K. O. and Vanderbilt, D., Structure and oxidation kinetics of the $\mathrm{Si}(100)-\mathrm{SiO}_{2}$ interface. Phys. Rev. B, in press.

[10] Tu, Y., Tersoff, J., Grinstein, G. and Vanderbilt D. Properties of a continuous-randomnetwork model for amorphous systems. Phys. Rev. Lett. 81, 4899 (1998).

[11] Wooten, F., Winer, K. and Weaire, D. Computer generation of structural Models of 
amorphous Si and Ge. Phys. Rev. Lett. 54, 1392 (1985).

[12] Laughlin, R. B. and Joannopoulos, J. D., Effect of second-nearest-neighbor forces on the vibrations of amorphous $\mathrm{SiO}_{2}$. Phys. Rev. B 17, 2790 (1978).

[13] Renaud, G., Fuoss, P. H., Ourmazd, A., Bevk, J., Freer, B. S. and Hahn P. O. Native oxidation of the $\mathrm{Si}(100)$ surface: Evidence for an interfacial phase. Appl. Phys. Lett. 58, 1044 (1991).

[14] Banaszak Holl, M. M., Lee, S., and McFeely, F. R., Core-level photoemission and the structure of the $\mathrm{Si} / \mathrm{SiO}_{2}$ interface: A reappraissal. Appl. Phys. Lett. 65, 1097 (1994). 


\section{Figure Captions}

Fig. 1. Plan view of two $\mathrm{Si}_{-} \mathrm{SiO}_{2}$ interfaces. The last three layers of $\mathrm{Si}$ are shown in gold, with atoms further from the interface shown smaller. The first layer of $\mathrm{O}$ is shown in red. (a) Stripe phase, having $(2 \times 1)$ symmetry. (b) Check phase, having $\mathrm{c}(2 \times 2)$ symmetry.

Fig. 2. Total energy $E$, and number of interfacial bridge bonds, versus number of accepted Monte Carlo steps. The decrease in the energy each time a bridge bond forms illustrates their crucial role in giving a low interface energy.

Fig. 3. (a) Side view of $4 \times 4$ stripe phase ([110] projection). The Si and O atoms are represented by gold and red spheres respectively. Each arrow points to a row of oxygen atoms that form the bridges at the interface. Notice the substantial voids above each bridge

bond. (b) Energy of each atom versus its z coordinate. Red circles represent oxygen atoms and gold circles represent silicon atoms. The green line is the local energy per atom, averaged over 20 configurations (and over a z range of $\sim 1 \AA$ for smoothness).

Fig. 4. Same as Fig. 3, for interface between Si and tridymite. 
This figure "fig1.gif" is available in "gif" format from: http://arxiv.org/ps/cond-mat/9903424v1 
This figure "fig2.gif" is available in "gif" format from: http://arxiv.org/ps/cond-mat/9903424v1 
This figure "fig3.gif" is available in "gif" format from: http://arxiv.org/ps/cond-mat/9903424v1 
This figure "fig4.gif" is available in "gif" format from: http://arxiv.org/ps/cond-mat/9903424v1 